\title{
Mood Baroco, Avicenna's proof of it, and reasoning by means of models
}

\author{
Miguel López-Astorga \\ Institute of Humanistic Studies "Juan Ignacio Molina”, University of Talca, Chile. \\ Email: milopez@utalca.cl
}

Received November 11, 2017; Revised January 09, 2018; Accepted January 15, 2018; Published May o6, 2018.

\begin{abstract}
:
In several works, the mental models theory has been used in order to check whether or not some ancient logics are more related to the actual human reasoning than standard or classical logic. In this paper, I try to make further analyses in this direction. In particular, I address mood Baroco in Aristotelian logic and the proof of it by Avicenna. My conclusions refer to that, apart from the fact that it is true that the mental models theory can indicate the possible cognitive scope that can be attributed to Aristotelian logic, both the initial version of Baroco and Avicenna's proof of that mood can also be very useful research instruments to help us explore and develop some particular aspects of the mentioned theory.
\end{abstract}

Keywords: Aristotelian logic; Avicenna; Baroco; mental models; reasoning

\section{Introduction}

As it is known, there is a wide literature on the mental models theory. Merely some relatively current works supporting it can be, e.g., Hinterecker, Knauff, and Johnson-Laird, 2016; Khemlani, Lotstein, Trafton, and Johnson-Laird, 2015; Khemlani, Orenes, and Johnson-Laird, 2012, 2014; Quelhas and Johnson-Laird, 2017; Quelhas, Rasga, and Johnson-Laird, 2017; Ragni, Sonntag, and Johnson-Laird, 2016. That literature seems to show that the theory is correct or, at the very least, that it has a great potential to account for certain human intellectual abilities. Undoubtedly, this fact is suggesting, since it can lead to think, for instance, that we are beginning to find the real way human beings reason or make inferences. Of course, one might object to ideas such as this one and argue that the mental models theory is not the only reasoning theory at present, and that there are also other theories with more or less consistency with empirical data provided by experiments. However, if the general experimental results reported in the mentioned literature are taken into account, it appears that the advantage is for the mental models theory, as this last approach is the one that is apparently based on more empirical evidence.

In any case, this paper will not deal with this discussion. The following pages mainly focus on the possibilities ancient logics can give to analyze, review, and clarify some aspects of the mental models theory. This theory has already been related to ancient logics in several works. For example, if we only consider Aristotelian logic, we can say that it has been tried to prove that, if we assume the aforementioned theory, we must also suppose that all of the moods of all of the figures in this last logic have to be necessarily easy for the human mind (López-Astorga, 2016a). Clearly, papers in this direction are interesting because they allow reviewing the predictive

(c) AesthetixMS 2018. This Open Access article is published under a Creative Commons Attribution Non-Commercial 4.0 International License (http://creativecommons.org/licenses/by-nc/4.o/), which permits non-commercial re-use, distribution, and reproduction in any medium, provided the original work is properly cited. For citation use the DOI. For commercial re-use, please contact editor@rupkatha.com. 
potential of a logic such as the Aristotelian one and, at the same time, checking some of the predictions of the mental models theory. Nevertheless, more aspects of more ancient logics have been studied from the framework of this theory. Simply continuing with Aristotelian logic, it can also be stated that the relationships of opposition have been addressed in the same way, i.e., resorting to the mental models theory too, in order to detect which of such relationships would be hard to teach for a teacher (López-Astorga, 2016b). There is no doubt that works with these goals are relevant as well, because they can be useful both in pedagogy (they can indicate how to teach the mentioned relationships) and in cognition (again, they can reveal whether or not certain predictions of the mental models theory are right).

Thus, it is evident that more examples of studies based on the mental models theory dealing with ancient logics could be cited, since, as pointed out, there are more works similar to those that have been indicated and related to both other aspects of Aristotelian logic and other ancient logical systems. Nonetheless, the main aim of this paper is not to comment on such works, but only to deepen the links between Aristotelian logic and the mental models theory to a larger extent. And this is so because what I will try to show is the general structure of interesting experiments using schemata from that logic that could clarify or verify some crucial consequences drawn from the essential theses of the mental models theory.

In this way, to do that, I will mainly consider a mood in the second figure in Aristotelian logic: Barocho, or, if preferred, Baroco (this last name will be the one used throughout this paper). In particular, I will analyze both its structure and the proof of it based on Reductio ad Absurdum method given by Avicenna in al-Isārāt wat-tanbīhāt. Thus, I will start with the way the mental models theory deems the quantified sentences included in Baroco. Then, paying attention to arguments such as those of López-Astorga (2016a), I will propose some reasons why it would be relevant to carry out experiments with the structure of the mentioned mood and comment on the information that such experiments could offer with regard to both the way we make inferences and the mental models theory. Thirdly, I will review Avicenna's proof in order to explain why it can give further information on the manner the human mind works (especially, as it can be seen below, on the manner the negation of quantified sentences is understood by people) and hence on other key aspects related to that very theory. However, as said, I begin with the mental models theory and its account about certain quantified sentences.

\section{The mental models theory and the sentences of type $A$ and $O$}

As it can be checked in papers such as that of Khemlani et al. (2015), the mental models theory provides accounts on a number of kinds of quantified sentences. Nevertheless, the only ones that are interesting here are those that are used in Baroco, that is, the sentences of kind A, i.e., the universally quantified affirmative sentences, and the sentences of kind O, i.e., the particularly quantified negative sentences. To understand the account of the mental models theory of these types of sentences, firstly, it is necessary to explain two important points of its general framework. On the one hand, the theory proposes that people usually think about sentences considering possible cases that can be consistent with them. On the other hand, such cases are not always detected without difficulties: some of them require minimal cognitive effort and others need more cognitive effort (see also, e.g., López-Astorga, 2016a). In this way, a set of cases requiring little cognitive effort is called by Khemlani et al. (2015) a 'canonical model', and a set of cases needing further effort is called by them a 'noncanonical model', their main prediction in this way being that most of the individuals will tend to consider only canonical models when reasoning, since 
these last models are, as said, easier. But how all of this applies to A and O sentences can be better seen by means of an example.

Let us think about the famous sentence used by Hempel (1945) to present his paradox of the black ravens, which is also taken by Nickerson:

[1] “All ravens are black" (Nickerson, 1996, p. 2).

Obviously, [1] is an A sentence, and what the mental models claims is that, when reflection about it, people, in principle and without much cognitive effort, often take into account only three possible cases compatible with it and akin to the following (see Khemlani et al., 2015, p. 2077, Table 1; see also, e.g., López-Astorga, 2016a, pp. 6-7):

[2] (Raven \& Black)

(Raven \& Black)

(Raven \& Black)

[2] is a canonical model of [1] and represents the cases of three different beings, which are all both ravens and black. Nonetheless, people can spend further effort, think about more possibilities, and come to this noncanonical model of [1] (see khemlani et al., 2015, p. 2077, Table 1; see also, e.g., López-Astorga, 2016a, pp. 7-8):

[3] (Raven \& Black)

(Not-raven \& Black)

(Not-raven \& Not-black)

Now, it has been noted that more cases are possible. Thus, the first element continues to stand for a being that is both raven and black. However, the second element is a being that, although it is black, it is not a raven. Finally, in the third case, we have a being that is neither raven nor black. Evidently, these two last possibilities are consistent with [1] too, but, as stated, it cannot be noted without effort.

As far as $\mathrm{O}$ sentences are concerned, it is easy to build one of them with concepts akin to (although not identical to) those of [1]:

[4] Some birds are not black.

Given a sentence such as this one, the mental models theory can lead us to a canonical model similar to the following (see Khemlani et al., 2015, p. 2077, Table 1; see also, e.g., López-Astorga, 2016a, p. 9):

[5]

(Bird \& Not-black)

(Bird \& Not-black)

(Not-bird \& black)

That is, a set with three beings in which the two first elements correspond to animals that are birds but not black, and the third one refers to a being that is not a bird but it does be black.

Nevertheless, in the same way as in the case of the A sentences, cognitive effort can help us come to a noncanonical model, which can be as follows (see Khemlani et al., 2015, p. 2077, Table 1; see also, e.g., López-Astorga, 2016a, p. 9): 
(Bird \& Not-black)

(Not-bird \& Black)

(Not-bird \& Black)

Clearly, the elements are now four, the first one and the second one being birds that are not black, and the third one and the fourth one being beings other than birds that, nonetheless, are black.

As indicated, the mental models theory, and, in particular, Khemlani et al. (2015), analyzes more types of quantified sentences. However, as also mentioned, A and $\mathrm{O}$ are the only types that are necessary if the aims of this paper are considered. Thus, I think that, to continue, it is only relevant to insist that the basic framework of the mental models theory about quantified sentences has already been used to review ancient logics with different goals. One of those goals have been, for example, as said too, to check whether or not all of the moods in Aristotelian logic are easy for the human mind, or, in other words, whether or not all of these moods require only canonical models to verify their conclusions (López-Astorga, 2016a). On the other hand, other of those goals has been, for instance, as also pointed out, to predict which relationships of opposition should be difficult to teach, that is, which of those relationships need noncanonical models to be understood (López-Astorga, 2016b). This is relevant because my arguments below are not very far from papers such as those ones. The reason of that is that I will focus, following López-Astorga's (2016a) analyses, on Baroco in the next section in order to show why, beyond what has been said by him, the revisal of that mood under the approach of the mental models theory can be interesting to better understand Aristotelian logic, that very theory, and cognition in general.

\section{Baroco and the mental models theory}

Indeed, my explanation in this section will be mainly based on López-Astorga's arguments, who in turn essentially follows Parson's (2008) chapter. Thus, it can be said that Baroco is a mood corresponding to the second figure, that is, the one that Aristotle accounts for in Analytica Priora I, 5, and that, therefore, the middle term is the predicate in its two premises. So, given this information along with the one that, as the name 'Baroco' reveals, its first premise is an A sentence, its second premise is an $\mathrm{O}$ sentence, and its conclusion is an $\mathrm{O}$ sentence as well, this example of the aforementioned syllogism can be built resorting to concepts such as those used in [1] and [4]:

[7] All ravens are black.

Some birds are not black.

Therefore, some birds are not ravens.

So, paying attention to both López-Astorga's (2016a) arguments and what has been commented on above, it can be stated that, while the canonical models of the first and the second premises are, respectively, [2] and [5], the combination of such models should be akin to this model:

[8] (Raven \& Black \& Not-bird)

(Raven \& Black \& Not-bird) 
(Raven \& Black \& Not-bird)

(......... \& Not-black \& Bird)

(......... \& Not-black \& Bird)

Obviously, what has been done here is, in a similar way as López-Astorga (2016a, pp. 20-21) does, to complete [2] with the information provided by [5]. Certainly, the two first elements of [5] has been added as the fourth and fifth elements in [8], and, at the same time, the information of the third element of [5] has allowed completing the three first elements of [8] coming from [2], in which, because they include the characteristic 'Black', 'Not-bird' has been included.

However, the problem is that, as it can be noted, the two last elements in [8], that is, its fourth and fifth elements, are incomplete, since they give no information on whether or not the birds indicated in them are ravens. Nevertheless, according to López-Astorga (2016a, p. 21), the solution of problems of this kind is very easy. Given that individuals know, by virtue of the first premise, that a being must have the property corresponding to the middle term (in this case, 'Black') if that being also have the property corresponding to the predicate of the conclusion, or the subject of the first premise (in this case, 'Raven'), they can 'update' a model such as [8] in a way akin to this one:

[9] (Raven \& Black \& Not-bird)

(Raven \& Black \& Not-bird)

(Raven \& Black \& Not-bird)

(Not-raven \& Not-black \& Bird)

(Not-raven \& Not-black \& Bird)

But, having reached this point, two important remarks must be made. Firstly, [9] would not be the real canonical model people would take into account in this case. The mental models theory predicts modulation processes as well (e.g., Quelhas et al. 2017). Such processes enable to block or remove possibilities that are semantically or pragmatically inconsistent, and this is clearly the case of the three first elements in [9], which are ravens and not birds. Obviously, given that general knowledge informs to us that all ravens are birds, those three elements have to disappear, the real structure of [9] being, for example,

[9b] (Not-raven \& Not-black \& Bird)

(Not-raven \& Not-black \& Bird)

Nevertheless, I do not think that this is an actual problem, since the conclusion is true in both cases (in both of them, [9] and [9b], there are two beings that are birds but not ravens). What is so, and this is already the second remark, is that maybe the step from [8] to [9], or, better still, [9b], is not easy and it is not possible if only canonical models are considered. Indeed, to note that the assertion 'all ravens are black' is incompatible with cases of ravens not being black, it is necessary to come to a noncanonical model such as [3], which shows all of the possibilities coherent with the mentioned sentence (Raven \& Black; Not-raven \& Black; Not-raven \& Notblack) and hence, although it is in an indirect way, that the only combination that is not allowed is the one in which the being is a raven and is not black.

This is a really important point, as, if what has been said in the previous paragraph is correct, that means that an individual can only note that Baroco is a right inference (or, in the case of my particular example, that the conclusion 'some birds are not ravens' follows the premises) if he/she 
spends certain cognitive effort. The conclusion states that there are beings that are not ravens and, according to the mental models theory, to be able to consider beings of this kind, at least, a mental process such as the one indicated above, which leads from [8] to [9b], must be made. Hence, to check that Baroco is a correct inference, canonical models are not enough. At a minimum, a noncanonical model is necessary, and this has a clear consequence in the general framework of the theory: that task is difficult for an average individual, since, as said, noncanonical models are not detected in a easy, direct, and quick way. Reflection is required and, because of it, it can be thought that Aristotelian logic is not actually as evident for the human mind as assumed in previous studies. Baroco and other syllogisms that, according to LópezAstorga (2016a), need similar updates can be hard.

But this can lead us to think about an interesting experimental study. True, if we want to verify the idea, held by the mental models theory, that noncanonical models require effort, we can carry out an experiment with the structure of Baroco in which the premises are given and the participants are asked to indicate whether or not the conclusion follows. If the mental models theory is accurate, most of them should not respond in a positive way, as, as explained, that would require to resort to noncanonical models and, as also pointed out, what is expected by the theory is that people tend to consider canonical models exclusively. However, if a positive response is received, that could lead to very engaging analyses. Obviously, it could be thought that the theory has to be reviewed in order to check whether or not the distinction between canonical and noncanonical models is as clear and categorical as the theory proposes. Nevertheless, it could also be supposed that the contents and the meanings of the words used in the hypothetical experiment could cause interferences. As indicated, semantics plays a relevant role in the mental models theory and, in the same way as modulation can block possibilities, it can reveal some of them that are implicit or hidden as well.

So, it is absolutely evident that such an experiment would be beneficial. It could confirm some theses of the theory or, if this were not the case, it would give the opportunity to review or explore certain points of that framework that, as far as I know, could be more developed. Furthermore, experimental tasks based on Baroco can provide even more information on cognition and the mental models theory. That is obvious if we consider the proof of it presented by Avicenna using Reductio ad Absurdum method. The next section is devoted to this issue.

\section{Avicenna's proof of Baroco and mental models}

It is well known that Avicenna was a Persian philosopher very interested, among other subject matters, in Aristotelian logic. Nonetheless, the aspect of his thought that is relevant for this paper is just the proof of Baroco he gives in his book al-Isārāt wat-tanbīhāt. As said, he offers his proof resorting to Reductio ad Absurdum method, but what is most important of it is that it can show the structure of another experimental task that can provide further information on the problems I am dealing with.

As Street (2004) reminds, Avicenna's system is characterized by certain distinctions and rules. However, as pointed out, I will only pay attention here to the mentioned proof. Street (2004, p. 547) also indicates its general structure, which basically consists of negating the conclusion with the intention of coming to a contradiction (that is, of what is habitually made when Reductio ad Absurdum is applied). In the case of my example above, that means to consider the sentence 'some birds are not ravens' to be false, and this leads us to another aspect of the mental models theory that is not developed enough yet: the way quantified sentences are denied. 
Following Street, how to do that is clear for Avicenna, since "When is not the case that some Js are not Bs, then all Js are Bs" (Street, 2004, p. 547; italics in text). Thus, the negation of the conclusion of my example would have to be 'all birds are ravens'. Nevertheless, this equivalence is not totally clear in the general framework of the mental models theory. As far as I know, the theory has only addressed the way propositional sentences are negated (e.g., in Khemlani et al., 2012, 2014). That way is very simple, since it provides that, when individuals deny a sentence, they usually consider the complement of the possibilities included in its model. For example, given a model consisting of a set with these elements:

[10] (A \& B)

(A \& Not-B)

The negation would be obtained just thinking about the missing elements:

[11]

(Not-A \& B)

(Not-A \& Not-B)

But, as said, this is the manner to negate assertions when they are propositional. It is true that in papers such as that of López-Astorga (2016b) the cases in which certain quantified sentences can be false have been considered from a point of view based on the mental models theory. Nonetheless, the general perspective in such works is very different from the one should be adopted here. In them, the focus is on pedagogy. In particular, as mentioned, in López-Astorga (2016b), the focus is on how to teach the relationships of opposition and how a teacher would have to work to show to his/her students which the real possibilities of a negated sentence are, and not on the way people naturally and swiftly deny quantified sentences. So, the only alternative seems to be to assume, as a working hypothesis (which, as shown below, can be very productive), that the way corresponding to propositional sentences applies to the quantified ones too. Thus, we can suppose that, when an individual denies the sentence 'some birds are not ravens', he/she thinks about the possibilities corresponding to the complement of its model. In this manner, given that the canonical model of this last sentence is clearly as follows:

[12] (Bird \& Not-raven)

(Bird \& Not-raven)

(Not-bird \& Raven)

Apart from the fact that the third element would be eliminated by modulation, the model corresponding to its negation would be this one:

[13] (Bird \& Raven)

(Not-bird \& Not-raven)

Since these possibilities are those that are missing in [12].

And, from this point on, the problem would be solved, as it seems that it is not difficult to conclude that 'all birds are ravens' from [13], the reason of that being that there is no bird that is not a raven in it. Furthermore, as in the version of Avicenna's proof given by Street (2004, p. 547), the problem is not the first premise, since [13] is compatible with its canonical model. In fact, if we combine [2] with [13], we obtain something similar to:

[14] (Bird \& Raven \& Black) 
Clearly, the second element of [13] has been removed in [14] because it is inconsistent with the three elements of [2]. Nonetheless, the combination with the canonical model of the second premise, that is, with [5], is impossible. In two of the possibilities of this last model, the bird is not black, which is incoherent with the only element of [14], and, in the other one, the being is not a bird, which is also incompatible with that very element.

Hence, as in Avicenna's proof, the key is the second premise. In that proof, that premise is “...some Js are not Ds..." (Street, 2004, p. 547; italics in text), and the negation of the conclusion leads to "...all Js are Ds" (Street, 2004, p. 547; italics in text). However, what is interesting about that is that an experiment akin to that described in the previous section and that were different from it in just the question, which would be now whether or not the denial of the conclusion follows, could provide a great deal of information as well. Certainly, only a clearly negative response would indicate, under the framework of the mental models theory, or, if preferred, of the interpretation of that theory provided here, that it is easy for people to deny quantified sentences, that is, to identify the complement of their canonical models. Otherwise, it could be thought that that intellectual action is generally hard to do.

Nevertheless, it is also possible to think in another experiment with even more informative potential. Indeed, an experiment with a structure that, although it is not exactly the same as the one of experiments such as the first one in Khemlani et al. (2014), is similar to it could be proposed in this direction too. In that experiment, which could be simply a condition of the previous one as well, it could be stated that 'some birds are not ravens' is a false sentence, and then asked which of these possibilities is/are compatible with that fact:

$$
\begin{aligned}
& \text { a.- There are birds that are ravens. } \\
& \text { b.- There are birds that are not ravens. } \\
& \text { c.- There are beings that are not birds and are ravens. } \\
& \text { d.- There are beings that are neither birds nor ravens. }
\end{aligned}
$$

There is no doubt that, if the previous approach based on the mental models theory is correct, the selection of alternatives a and $d$ in [15] would reveal that it is true that people tend to negate quantified sentences considering the complement of their canonical models, and that that is a very simple and easy activity for them. Of course, as the theory proposes, it is always possible that an individual reflects to a greater extent and comes to a noncanonical model, which, obviously, would be denied taking into account the complement of that noncanonical model, and, evidently, not the one of the canonical model. However, it would be expected that the habitual and more frequent responses were related to canonical models. In any case, an aspect that should be considered in any circumstance is the possible interferences that can be caused by general knowledge. As pointed out above, the mental models theory acknowledges the existence of modulation processes on which the semantic contents and the meanings of the words that appear in the inferences can have an influence. For this reason, perhaps it can be more suitable to use more abstract materials in experiments of this kind and resort in them to, instead of well known concepts such as 'raven', 'bird', or 'black', expressions such as those used in the version of the proof by Avicenna presented in Street (2004), that is, expressions such as 'Js', 'Bs', or 'Ds'.

Undoubtedly, if the experiment is thought in this way, while it would be more complex, it would provide a clearer information on the general way quantified sentences are usually negated, since facts very known by people, such as that many birds are not ravens or that, if an animal is not a 
bird, it cannot be a raven either, would not make it more difficult to identify the actual underlying inferential activity. Therefore, there is something that is undisputable: if an experiment of this kind is carried out, the task or tasks included in it must be carefully built. Otherwise, we will not be sure whether or not it gives us real relevant information on the mental models theory, cognition, and although it is in an indirect manner, Aristotelian logic.

\section{Conclusions}

All of the conclusions I will present in this last section are, for obvious reasons, very akin to conclusions raised in papers such as, for example, those of López-Astorga (2016a, 2016b). It is worth resorting to the mental models theory to analyze ancient logics because, as said, it has a strong empirical support. I have cited above only some of the studies addressing it, but there are many more papers, chapters, books... in the same direction. Most of them report empirical or experimental researches and almost all of them show results that confirm the basic theses of the theory.

Hence, it is certainly interesting to use it in studies linked to such logics. Besides, as LópezAstorga indicates, it can also be beneficial for the mental models theory. As shown, a particular aspect of an ancient logic such as the Aristotelian one can help us clarify dark points in the theory, develop it to a greater extent, and better understand its consequences and what it implies, as well as reveal some supposition of it that needs to be reviewed. So, it is obvious that it would be very productive for the proponents of the aforementioned theory to work in directions such as the one indicated in this study, that is, resorting to schemata provided in ancient times to, considering the contents that are deemed to be suitable, carry out experiments at present.

However, returning to the beginning of this section and always in keeping with conclusions similar to the ones of López-Astorga (2016a, 2016b), it can also be said that activities of that type can be relevant for ancient logics as well. Such activities can allow discovering their real status and what they actually described. Of course, it is highly questionable that Aristotle or Avicenna wanted, as the mental models theory does, to account for the way the human mind works. Nevertheless, analyses such as that presented in this paper can reveal that, beyond their real intentions and goals, it is possible that some ancient logical systems offered, at least partially, structures or kinds of inferences habitual for human beings, or that human beings often use without any difficulty. To work in this direction can be important in fields such as the one of knowledge theory or cognitive science too, since, as said, it can determine the real value and the actual scope of such logics. And, clearly, this can lead one to think about another key point claimed by López-Astorga as well: that the possibility exists that some ancient logics are closer to the mental models theory than standard logic as understood nowadays. Given the arguments presented above, this last possibility undoubtedly deserves to be taken into account. Therefore, it is a line that it is also worth continuing to explore.

\section{References}

Hempel, C. G. (1945). Studies in the logic of confirmation (I). Mind, 54, 1-26.

Hinterecker, T., Knauff, M., \& Johnson-Laird, P. N. (2016). Modality, probability, and mental models. Journal of Experimental Psychology: Learning, Memory, and Cognition, 42(10), 1606-1620. 
Khemlani, S., Lotstein, M., Trafton, J. G., \& Johnson-Laird, P. N. (2015). Immediate inferences from quantified assertions. The Quarterly Journal of Experimental Psychology, 68(10), 2073-2096.

Khemlani, S., Orenes, I., \& Johnson-Laird, P. N. (2012). Negation: A theory of its meaning, representation, and inference. Psychological Review, 109(4), 646-678.

Khemlani, S., Orenes, I., \& Johnson-Laird, P. N. (2014). The negation of conjunctions, conditionals, and disjunctions. Acta Psychologica, 151, 1-7.

López-Astorga, M. (2016a). Does Aristotelian logic describe human reasoning? Valid syllogisms and canonical models. Khazar Journal of Humanities and Social Sciences, 19(2), 5-27.

López-Astorga, M. (2016b). The logical relations of opposition and how to teach them. Opción, 32(79), 3249.

Nickerson, R. S. (1996). Hempel's paradox and Wason's selection task: Logical and psychological puzzles of confirmation. Thinking and Reasoning, 2, 1-31.

Parsons, T. (2008). The development of supposition theory in the later $12^{\text {th }}$ through $14^{\text {th }}$ centuries. In D. M. Gabbay \& J. Woods (Eds.), Handbook of the History of Logic. Volume 2. Mediaeval and Renaissance Logic (157-280). Amsterdam, The Netherlands: Elsevier.

Quelhas, A. C. \& Johnson-Laird, P. N. (2017). The modulation of disjunctive assertions. The Quarterly Journal of Experimental Psychology, 7o(4), 703-717.

Quelhas, A. C., Rasga, C., \& Johnson-Laird, P. N. (2017). A priori true and false conditionals. Cognitive Science, 41(55), 1003-1030.

Ragni, M., Sonntag, T., \& Johnson-Laird, P. N. (2016). Spatial conditionals and illusory inferences. Journal of Cognitive Psychology, 28(3), 348-365.

Street, T. (2004). Arabic logic. In D. M. Gabbay \& J. Woods (Eds.), Handbook of the History of Logic. Volume 1. Greek, Indian and Arabic logic (523-596). Amsterdam, The Netherlands: Elsevier. 